\title{
ON BOUNDARY REGULARITY FOR PLATEAU'S PROBLEM'
}

\author{
BY WILLIAM K. ALLARD
}

Communicated by Herbert Federer, November 15, 1968

We state here sufficient conditions for certain minimal surfaces to be differentiable at boundary points.

Let $m$ and $n$ be integers with $1<m<n$. We adopt the notation of [3]. See also [2]. In particular, $I_{m}\left(R^{n}\right)$ is the group of $m$ dimensional integral currents in $R^{n}$. If $T \in I_{m}\left(R^{n}\right), M(T)$ is the mass of $T$ and $\partial T$ is the boundary of $T$; if $a \in R^{n}, \Theta^{m}(\|T\|, a)$ is the $m$ dimensional density of the variation measure $\|T\|$ at $a$.

If $T \in I_{m}\left(R^{n}\right)$, we say $T$ is minimal if there exists $r>0$ such that $M(T) \leqq M(S+T)$ whenever $a \in R^{n}, S \in I_{m}\left(R^{n}\right), \partial S=0$ and spt $S$ $C\{x:|x-a|<r\}$. Given $B \in I_{m-1}\left(R^{n}\right)$ with $\partial B=0$, it is shown in [3] that there exists $T \in I_{m}\left(R^{n}\right)$ such that $\partial T=B$ and $M(T)$ $\leqq M(S+T)$ whenever $S \in I_{m}\left(R^{n}\right)$ with $\partial S=0$.

Theorem. Suppose $T \in I_{m}\left(R^{n}\right), T$ is minimal, $a \in$ spt $\partial T, p \geqq 2$, $\Theta^{m-1}(\|\partial T\|, a)=1$ and spt $\partial T$ intersects some neighborhood of $a$ in $a$ class $p$ (real analytic) $m-1$ dimensional submanifold of $R^{n}$.

(1) If $\Theta^{m}(\|T\|, a)=1 / 2$, then the intersection of spt $T$ with some neighborhood of $a$ is a subset of some class $p-1$ (real analytic) $m$ dimensional submanifold of $\boldsymbol{R}^{n}$.

(2) If there exist independent linear functionals $\alpha_{i}, i=1, \cdots$, $n-m+1$, on $R^{n}$ such that either

$$
\operatorname{spt} \partial T \subset\left\{x: \alpha_{i}(x-a) \geqq 0, i=1, \cdots, n-m+1\right\},
$$

or there is $r>0$ such that

$$
\begin{aligned}
\{x:|x-a|<r\} \cap \operatorname{spt} T \subset\left\{x: \alpha_{i}(x-a)\right. & \geqq 0, \\
& i=1, \cdots, n-m+1\},
\end{aligned}
$$

then $\Theta^{m}(\|T\|, a)=1 / 2$.

Corollary. Suppose $p \geqq 2$ and $B$ is the $m-1$ dimensional integral current corresponding to some compact oriented class $p$ (real analytic) $m-1$ dimensional submanifold $N$ of $R^{n}$. If $N$ lies on the boundary of some uniformly convex open subset of $R^{n}$ and $T \in I_{m}\left(R^{n}\right)$ is minimal

${ }^{1}$ This work was partially supported by the National Science Foundation, and part of it is contained in the author's doctoral thesis at Brown University. 
with $\partial T=B$ then there is $r>0$ and a class $p-1$ (real analytic) $m$ dimensional submanifold $M$ of $\boldsymbol{R}^{n}$ such that

$$
\text { spt } T \cap\{x \text { : distance }(x, N)<r\} \subset M \text {. }
$$

Statement (1) is proved by combining the interior regularity results of [1] or of [8] with the construction of certain surfaces of dimension $n-1$ which are barriers for the $m$ dimensional area problem, and then applying the higher differentiability results of [6] and [7]. Statement (2) is proved by applying a variational argument to a tangent cone of $T$ at $a$. The corollary is an elementary consequence of the theorem.

These results remain true if we replace the group $I_{m}\left(R^{n}\right)$ by the group of flat chains over the integers modulo 2, as in [5]. If $m=2$ and $n=3$, the only boundary density that occurs on the smooth boundary of a minimal chain is one half. In view of the interior regularity results of [4], a minimal flat chain over the integers modulo 2 in $R^{3}$ which spans a finite family of smooth curves must be free from singularities of any kind.

I gratefully acknowledge the encouragement and advice of Professors Herbert Federer and Wendell Fleming and the substantial assistance given me by Professor Frederick J. Almgren, Jr.

\section{REFERENCES}

1. F. J. Almgren, Jr., Existence and regularity almost everywhere of solutions to elliptic variational problems among surfaces of varying topological type and singularity structure, Ann. of Math. (2) 87 (1968), 321-391.

2. H. Federer, Geometric measure theory, Springer-Verlag, New York, 1969.

3. H. Federer and W. H. Fleming, Normal and integral currents, Ann. of Math. (2) 72 (1960), 458-520.

4. W. H. Fleming, On the oriented Plateau problem, Rend. Circ. Mat. Palermo (2) 11 (1962), 69-90.

5. - Flat chains over a finite coefficient group, Trans. Amer. Math. Soc. 121 (1966), 160-186.

6. C. B. Morrey, Jr., Second order elliptic systems of differential equations, Ann. of Math. Studies No. 33, Princeton Univ. Press, Princeton, N. J., 1954, pp. 101-159.

7. - On the analyticity of solutions of analytic non-linear elliptic systems of partial differential equations. I, II, Amer. J. Math. 80 (1958), 198-218, 219-234.

8. E. R. Reifenberg, An epiperimetric inequality related to the analyticity of minimal surfaces, Ann. of Math. (2) 80 (1964), 1-14.

Brown University, Providence, Rhode Island 02912 and

Princeton University, Princeton, New Jersey 08540 